\title{
Cloud Fractions Estimated from Shipboard Whole-Sky Camera and Ceilometer Observations
}

\author{
By Makoto KujI, ${ }^{1)}$ Risa Fusimoto, ${ }^{1)}$ Mayu Miyagawa, ${ }^{1)}$ Ryoko Funada, ${ }^{1)}$ Masahiro Hori, ${ }^{2)}$ Hiroshi Kobayashi, ${ }^{3)}$ Seizi \\ KoGA, ${ }^{4)}{ }^{\prime}$ Junji MatsushitA ${ }^{5)}$ and Masataka SHIOBARA ${ }^{5)}$ \\ ${ }^{1)}$ Graduate School of Humanities and Sciences, Nara Women's University, Nara, Japan \\ 2) JAXA, Tsukuba, Japan \\ 3) University of Yamanashi, Kofu, Japan \\ ${ }^{4)}$ AIST, Tsukuba, Japan \\ ${ }^{5)}$ NIPR, Tachikawa, Japan
}

(Received July 31st, 2015)

\begin{abstract}
Shipboard observations of cloud fraction were performed along the round-trip research cruise between Japan and Antarctica from November 2013 to April 2014 using both a whole-sky camera and a ceilometer onboard the Research Vessel (R/V) Shirase. Based on the results of the comparison of these cloud fractions, it was found that the correlation coefficient was 0.86 for the 2024 observations acquired between Australia and Antarctica from 27 November to 9 December 2013. Overall, the results were consistent between the two observation methods; however, some underestimation by the whole-sky camera was determined in comparison with the ceilometer. It is intended that shipboard observations will continue onboard the R/V Shirase over subsequent every years in order to improve the retrieval of cloud fractions for the validation of satellite observations.
\end{abstract}

Key Words: Second-Generation Global Imager (SGLI), Validation, Cloud, Whole-Sky Camera, Ceilometer

\section{Introduction}

Clouds play important roles in the earth's climate. They reflect solar radiation that leads to a cooling effect, but they also absorb infrared radiation and re-emit it to earth's surface, which causes warming. The cloud fraction is one of the fundamental properties that control these cooling and warming effects.

The Japan Aerospace Exploration Agency (JAXA) has a plan to launch an earth-observing satellite under the Global Change Observation Mission - Climate (GCOM-C). The GCOM-C satellite will carry a second generation global imager (SGLI), that is intended to retrieve cloud fraction as a standard atmospheric product.

Generally, it is important to validate satellite products using ground-based observations. However, oceans cover about $70 \%$ of the earth's surface and they have few observational sites. Therefore, shipborne observations are very important, even though they tentd to be relatively limited in coverage and generally suffer because of ship movement.

As for aerosol observation, National Aeronautics and Space Administration (NASA) deploys Microtops handheld Sun photometers globally under the Maritime Aerosol Network (MAN), which is a component of the Aerosol Robotic Network (AERONET). 1)

Recently, the aerosol properties over the western Mediterranean basin have been investigated using AERONET and MAN aerosol data. It was found that the properties retrieved by MAN were surprisingly similar to those obtained by the three nearest AERONET sites. ${ }^{2)}$

For the determination of cloud properties, German researchers performed the shipboard measurements using lidar and microwave remote sensing as well as spectral zenith ra- diance observations. They proposed a multispectral cloud retrieval method for shipboard measurements to obtain cloud optical thickness, effective particle radius, and liquid water path, based on three observational cruises at latitudes of between $50^{\circ} \mathrm{N}$ and $50^{\circ} \mathrm{S}$ over the Atlantic Ocean as part of the German Leibniz-network OCEANET project. ${ }^{3)}$

In this study, we undertook shipboard observations using both a whole-sky camera and a ceilometer to obtain cloud fractions. We describe the observations in detail in section 2. The cloud detection method is described in section 3 . The data analyses and the results are shown in section 4 . We privide a discussion on the methodology and the results in section 5. Finally, we summarize the preliminary results and describe a future perspective regarding shipboard observations in section 6 .

\section{Observation}

The Japanese Maritime Self-Defense Force operates the Japanese icebreaker (R/V) Shirase (AGB-5003), the length and displacement of which are $138 \mathrm{~m}$ and approximately 20,000 tons, respectively. The ship conducts a training cruise around Japan in September every year. Figure 1 shows the ship track during the training cruise in 2013.

$\mathrm{R} / \mathrm{V}$ Shirase also makes a round-trip cruise between Japan and Antarctica. Figure 2 shows the ship track during the annual Antarctic cruise. The ship travels from Japan to Antarctica via the west of Australia and it returns from Antarctica to Japan via eastern Australia. The entire journey takes from November to March or April of the following year.

A whole-sky camera and a ceilometer are included in the instrument package onboard R/V Shirase. We obtained sky images at 5-min intervals using the whole-sky camera, and we ac- 


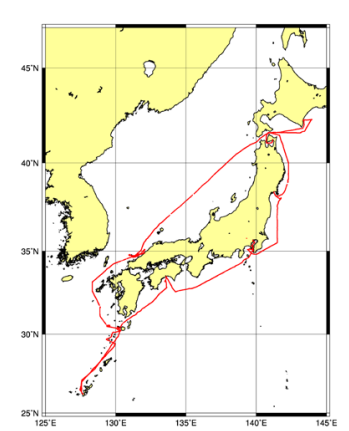

Fig. 1. Ship track around Japan from August to November 2013. $\mathrm{R} / \mathrm{V}$ Shirase travels anticlockwise.

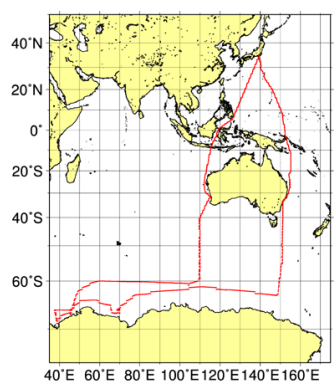

Fig. 2. Ship track between Japan and Antarctica from November 2013 to April 2014. R/V Shirase travels anticlockwise.

quired cloud bottom profiles at 36-s intervals using the ceilometer, during the cruise around Japan in the autumn 2013 and over the Pacific Ocean from November 2013 to April 2014. The details of the observational period are summarized in Table 1.

Table 1. Summary of the observational period along the R/V Shirase cruise during the 55th Japan Antarctic Regional Experiment (JARE 55). The observation event indicates the number of images and profiles obtained with the whole-sky camera and the ceilometer, respectively. We did not acquire any profiles with the ceilometer during the training cruise.

\begin{tabular}{ccr}
\hline Cruise & Observation period & Events \\
\hline Training & Whole-sky camera & \\
To Antarctica & 26 Aug. - 07 Nov. 2013 & 21229 \\
Syowa station & 05 - 10 Jan. 2014 & 1488 \\
From Antarctica & 10 Feb. - 08 Apr. 2014 & 16267 \\
\hline \multicolumn{3}{c}{ Ceilometer } \\
\hline Training & No operation & 0 \\
To Antarctica & 27 Nov. 2013 - 04 Jan. 2014 & 92159 \\
Syowa station & 05 - 21 Jan. 2014 & 40801 \\
From Antarctica & 22 Jan. - 07 Apr. 2014 & 180694 \\
\hline
\end{tabular}

Figure 3 shows the whole-sky camera system that was mounted on the uppermost deck of R/V Shirase. The whole-sky camera comprised a digital camera (NIKON D7000, NIKON Corp.) and a circular fisheye lens (4.5 mm F2.8 EX DC Circular Fisheye HSM, SIGMA Corp.) to make a photography of a whole sky. The system was covered with a cylindrical housing to protect it from sea spray and filled with silica gel to avoid condensation inside the housing, including the transparent dome at the top. The housing was raised above the deck by about $150 \mathrm{~cm}$ to obtain the widest view of the sky as possible.

The observed whole-sky images were transferred automatically to an archiving laptop PC at specified intervals. They were also backed up temporarily to a USB memory. The photography and data transfer were controlled with an Armadillo-440 board with a Linux operating system (Atmark Techno, Inc.). Each image consisted of 4928 pixels $\times 3264$ lines (e.g., Fig. 5a). The circular fisheye lens was equisolid angle type; thus, the number of cloudy pixels corresponded to the cloud amount observed by eye.

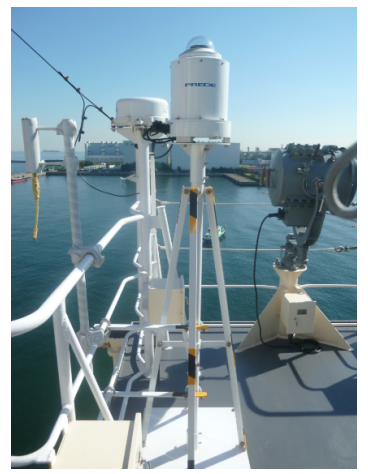

Fig. 3. Whole-sky camera system mounted on the uppermost deck of R/V Shirase.

A ceilometer (Vaisala CL51) was also mounted several meters from the whole-sky camera as shown in Fig. 4. The ceilometer provided measurements of cloud bottom height at 36-s intervals throughout the cruises, from which the cloud fraction could be estimated as an appearance frequency. The vertical resolution was $10 \mathrm{~m}$ and the uppermost altitude was 15 $\mathrm{km}$. The laser wavelength was $910 \mathrm{~nm}$. Vaisala's operational algorithm provided up to three cloud bottom heights from the surface.

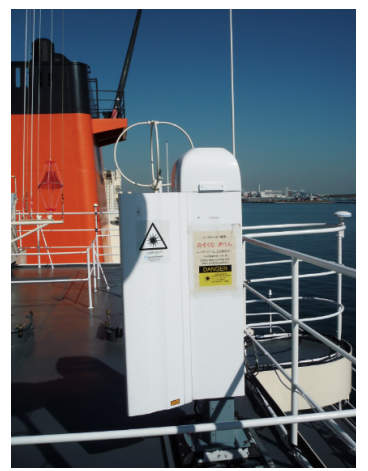

Fig. 4. Ceilometer system (Vaisala CL51) mounted on the uppermost deck of R/V Shirase near the whole-sky camera. The side cover is shown open for maintenance.

\section{Cloud Detection Method}

In this section, we describe the preprocessing of the wholesky images and the retrieval algorithm of the cloud detection method. 


\subsection{Preprocessing}

The image obtained from the whole-sky camera was converted to a JPEG format, as shown in Fig. 5. Preprocessing of the original JPEG image is necessary because of many black pixels surrounding the sky view. Furthermore, the round edge was trimmed for sun elevations of less than $10^{\circ}$ because of the influence of the surface albedo on sky brightness and the spectral features. We also confined the pixels in the image to a zenith angle of less than $70^{\circ}$ to avoid interference from surface structures such as masts and to suppress the influence of the sea surface due to ship fluctuation. The red circle in Fig. 5a shows the zenith angle of $70^{\circ}$ and the yellow square is a circumscription. As a result, the original observed JPEG image in Fig. 5a was trimmed as shown in Fig. 5b. The trimmed image comprised 2228 pixels $\times 2228$ lines; thus, the data volume was reduced to about $30 \%$ of the original JPEG image.

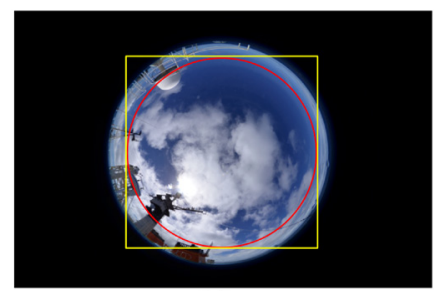

(a)

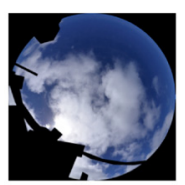

(b)
Fig. 5. Example image of the whole-sky camera. (a) Original JPEG image and (b) trimmed image on 06:15:19 UTC 1 December 2013. The red circle corresponds to the view angle of $70^{\circ}$ from the zenith and the yellow square is a circumscription. The top and right directions of the image correspond to the head and left direction of R/V Shirase, respectively.

There are a number of algorithms available to detect clouds in the images from a whole-sky camera.

As shown in Ref. 4), an automatic cloud classification algorithm was developed based on a set of mainly statistical features that describe the color as well as the texture of an image. A knearest-neighbor classifier was used because of its high performance in solving complex issues, simplicity of implementation, and low computational complexity.

As shown in Ref. 5), a method was proposed to retrieve cloud fraction from whole-sky images obtained at the polar regions, and the method was applied to the whole-sky camera system at Ny-Ålesund. The retrieved cloud fraction was validated using Micro-Pulse Lidar (MPL) and it was found consistent.

We analyzed the images and estimated cloud fractions in each image based on the algorithm using brightness and spectral information ${ }^{6)}$ as explained in the following subsections.

\subsection{SI-BI index}

Usually, we see a clear sky as blue and clouds as white or gray in the images obtained with the whole-sky camera. Therefore, a clear sky has high spectral contrast, i.e., larger Blue values and smaller Red values in the $R G B$ color images obtained with a digital camera that covers the visible spectral region. However, clouds have low spectral contrast among the Blue, Green, and Red values. On average, whiter clouds have brighter values in the visible spectral region, while grayish clouds have darker values.
Based on the fundamental features of the light spectrum of the whole-sky images, we can define a Sky Index $S I$ that is sensitive to the sky color, i.e., blue or white, using Eq. (1). Furthermore, we can also estimate the sky brightness using a Brightness Index $B I$, as defined in Eq. (2).

$$
\begin{gathered}
S I=\frac{\text { Blue }- \text { Red }}{\text { Blue }+ \text { Red }}, \\
B I=\frac{\text { Red }+ \text { Green }+ \text { Blue }}{255 \times 3},
\end{gathered}
$$

where Red, Green, and Blue are the digital numbers corresponding to Red, Green, and Blue, and those numbers range from 0 to 255; i.e., they are capable of expressing 256 levels.

$S$ I ranges from -1 to 1 : larger positive values correspond to a pure blue sky, zero corresponds to white cloud, and larger negative values correspond to pure reddish sky, such as a morning or evening glow.

$B I$ ranges from 0 to 1 : larger and smaller values correspond to brighter and darker regions, respectively. It should be noted that regions at and around the sun correspond approximately to $(S I, B I)=(0,1)$.

\subsection{Classification curve}

When we make a scatter plot of the $S I-B I$ domain, we generally have two distinctive clusters corresponding to cloud and clear sky regions in the whole-sky image. We show an example of a scatter plot based on data obtained at 06:15:19 UTC on 1 September 2013 in Fig. 6.

Furthermore, we can perform curve fitting on both clusters using the exponential function in Eq. (3).

$$
B I=e^{-k \times S I},
$$

where $k$ is the exponential coefficient of the classification curve.

Here, we may call the curves for the two clusters as the clear (blue) sky and cloud curves with coefficients $k_{b}$ and $k_{c}$, respectively. It is easily seen that we have a trough between the two clusters of clear sky and cloud. Thus, we can determine the classification curve with coefficient $k$ for the trough using a similar exponential function between the two curves fitted to the clear sky and the cloud.

\subsection{Determination of coefficient $k$}

At first, we selected the analysis data from 27 November 2013 14:00 UTC to 9 December 2013 13:30 UTC, during the period after the ceilometer operation was initiated and before $\mathrm{R} / \mathrm{V}$ Shirase moved into the sea ice region; i.e., we selected an open ocean region based on data obtained at sea ice. It is one of our future tasks to determine the value of coefficient $k$ in Eq. (3) over the sea ice region, because the higher surface albedo due to sea ice will affect both the sky brightness and the spectral contrast. We selected images with sun elevation of not less than $10^{\circ}$ because the surface albedo will increase considerably when the sun elevation is less than $10^{\circ}$ (cf. Fig. 11). As a result, we had 2043 images that satisfied the above criteria.

We chose 413 overcast images and 99 clear sky images through visual examination. We created the $S I-B I$ diagrams and performed curve fitting for each overcast or clear sky image so that we could determine the exponential coefficients $k_{c}$ and 


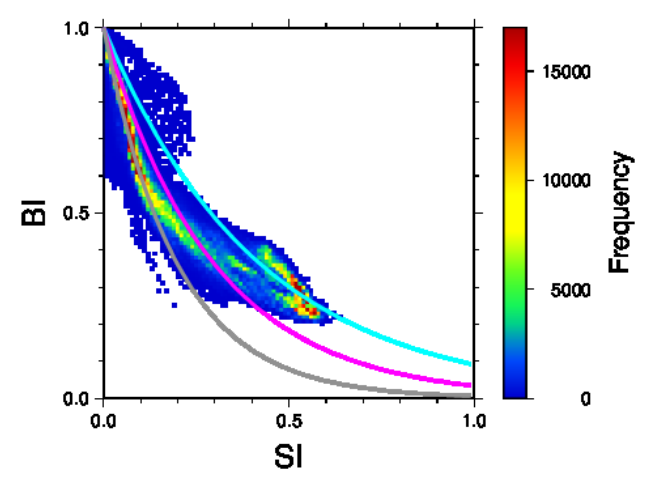

Fig. 6. Example classification diagram based on data obtained at 06:15:19 UTC on 1 December 2013 with coefficient $k=3.7$ in Eq. (3). The color code is the frequency, i.e., the number of the plots or pixels. The light blue and gray curves show the least square fits corresponding to a clear sky and cloud clusters, respectively. The pink curve is determined based on the average of the exponential coefficients for the clear sky and cloudy fits in Eq. (4).

$k_{b}$, respectively. It is easily seen that the maximum $k_{b}$ among the 99 clear sky images is the lowest curve on the $S I-B I$ diagram, while the minimum $k_{c}$ among the 413 overcast images corresponds to the highest curve. Coefficient $k$ of the classification curve should be between the minimum $k_{c}$ and the maximum $k_{b}$. Then, we can determine coefficient $k$ using Eq. (4):

$$
k=\frac{k_{b, \max }+k_{c, \min }}{2} .
$$

The maximum value of $k_{b}$ and the minimum value of $k_{c}$ were about 2.8 and 4.5, respectively, in this analysis. Consequently, we set the exponential coefficient $k$ at 3.7 using Eq. (4) in this preliminary analysis.

Using the classification curve, we can identify pixels in the image as either sun, cloud, or clear sky. When a pixel has a value of approximately unity in the brightness index $B I$, the pixel is identified as the solar disk, aureole, or adjacent brighter cloud. The remaining pixels should be classified as either cloud or clear sky in this scheme.

It is noted that coefficient $k$ of the classification curve in Eq. (3) does not have a universal value because the coefficient is possibly dependent on the diaphragm, attenuation of the filter, or sun elevation. The validity of the coefficient values, i.e., $k=$ 3.7 , is discussed later.

\section{Data Analyses and Results}

Based on the $S I-B I$ method described in the previous section, we classified the pixels as sun, cloud, or clear sky. The pixels for which $(S I, B I)$ equals $(0,1)$ correspond to the sun, whereas pixels above and below the classification curve correspond to clear sky and cloud, respectively. Figure 7 shows an image obtained by whole-sky camera and the classified result. The red, white, and blue pixels in Fig $7 \mathrm{~b}$ are sun, cloud, and clear sky, respectively. The cloud fraction (\%) of the whole-sky image $\left(C F_{\text {cam }}\right)$ is estimated as follows:

$$
C F_{\text {cam }}=\frac{N_{\text {CloudPixel }}}{N_{\text {AllPixel }}},
$$

where $N_{\text {CloudPixel }}$ and $N_{\text {AllPixel }}$ are the numbers of pixels of cloud and of the whole sky (i.e., sun, cloud, and clear sky pixels), respectively. As a result, the fraction was about $51 \%$ in this case.

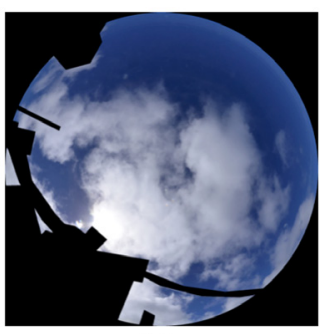

(a)

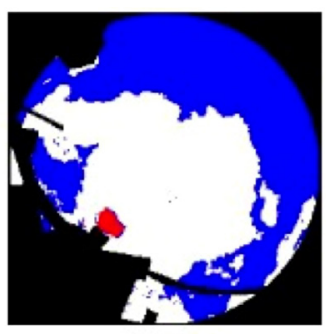

(b)
Fig. 7. Example image from the whole-sky camera. (a) Trimmed image and (b) classified image obtained at 06:15:19 UTC on 1 December 2013 with coefficient $k=3.7$ in Eq. (3). The red, white, and blue pixels correspond to sun, cloud, and clear sky, respectively.

Cloud bottom heights were derived using the Vaisala ceilometer CL51. We can define the cloud fraction (\%) of the ceilometer $\left(C F_{\text {ceil }}\right)$ for a given time bin (i.e., $5 \mathrm{~min}$ in this analysis), as follows:

$$
C F_{\text {ceil }}=\frac{N_{\text {CloudProfiles }}}{N_{\text {AllProfiles }}},
$$

where $N_{\text {CloudProfiles }}$ means the number of cloudy profiles for which cloud bottom heights were detected and $N_{\text {AllProfiles }}$ represents the number of available profiles between $\pm 2.5 \mathrm{~min}$ of the observation time of the whole-sky image.

We compared the spatial and temporal cloud fractions estimated from the whole-sky images and the ceilometer, respectively. Figure 8 shows a time series of cloud fractions on 29 November 2013 from Eqs. (5) and (6) for the whole-sky image and the ceilometer, respectively. It can be seen from Fig. 8 that the cloud fraction from the ceilometer had greater variation compared with the whole-sky camera during this period.

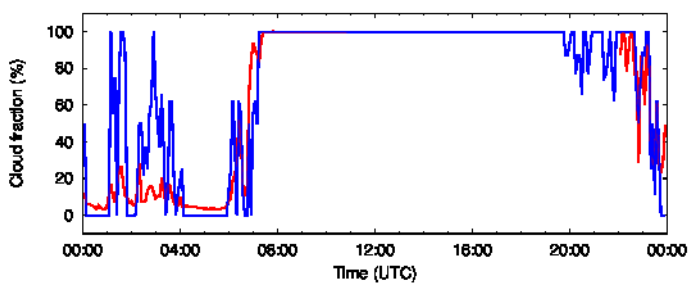

Fig. 8. Time series of cloud fractions from the whole-sky camera (red) and ceilometer (blue) on 29 November 2013. The cloud fraction from the whole-sky camera is not shown between 10:55 and 21:55 because the solar elevation was less than $10^{\circ}$.

Figure 9 shows a map of the cloud fractions estimated from the whole-sky camera (Fig. 9a) and the ceilometer (Fig. 9b) along the ship's track from 27 November to 9 December 2013. The plots correspond to an open ocean area where the value of coefficient $k=3.7$ in Eqs. (3) and (4) is applicable in this preliminary analysis. From Fig. 9, it can be seen clearly that the cloud fraction at latitudes lower than $60^{\circ}$ is generally smaller 
(a)

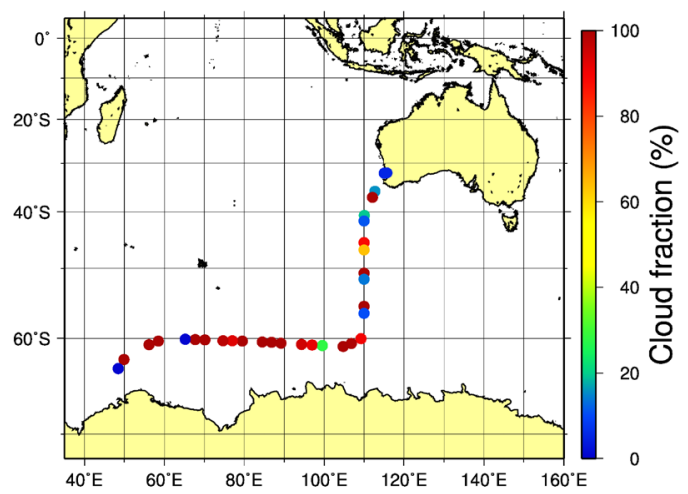

(b)

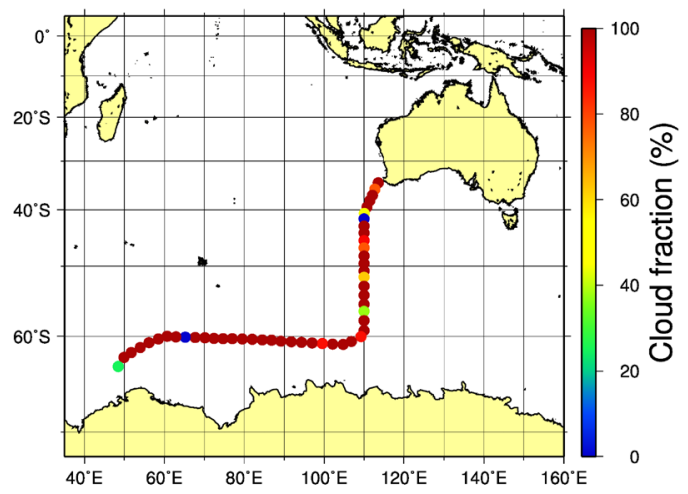

Fig. 9. Map of cloud fraction estimated from (a) the whole-sky camera and (b) the ceilometer between Australia and Antarctica from 27 November to 9 December 2013. The interval of plots is $6 \mathrm{~h}$ but there is a lack of plots at solar elevations lower than $10^{\circ}$ for the whole-sky camera (a).

than at higher latitudes for both the whole-sky camera and the ceilometer.

Figure 10 is a scatter plot of the cloud fractions between the whole-sky camera in Fig. 9a and the ceilometer in Fig. 9b between Australia and Antarctica from 27 November 2013 to 9 December 2013. The statistics are summarized in Table 2.

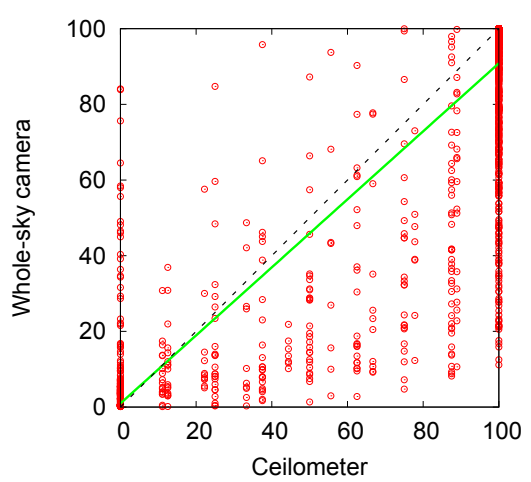

Fig. 10. Scatter plot of the cloud fractions from the whole-sky camera and the ceilometer. Green and black lines are the regression and one-to-one lines, respectively.

From Fig. 10 and Table 2, it can be established that the whole-sky camera generally underestimated the cloud fraction compared with the ceilometer. In addition, the correlation between the cloud fractions from the whole-sky camera and the
Table 2. Statistics of the comparison of cloud fractions between the whole-sky camera and the ceilometer, corresponding to Fig. 10.

\begin{tabular}{cc}
\hline Statistics & Values \\
\hline Regression line & $y=0.90 x+0.96$ \\
Correlation coefficient & 0.86 \\
RMSE & 18.4 \\
Number of data & 2024 \\
Deviation from one-to-one line & 20.1 \\
\hline
\end{tabular}

ceilometer is high (0.86). The regression line is close to a oneto-one representation; however, the deviation is approximately $20 \%$ and it increases in overcast situations. To minimize this deviation in the future, we could devise a comparison such that we make a match-up analysis with a temporal bin longer than 5 min and with a narrower spatial bin only in the zenith direction.

The whole-sky camera system in Ref. 7), was similar to this study, except it was mounted on the ground site. The cloud fraction estimated from the whole-sky camera based on the $S I-B I$ diagram ${ }^{6}$ was compared with that derived from MODIS observations. This study revealed that the comparison between the ground-based and satellite imager observations showed consistent assessments of the cloud fractions. As in Ref. 8), the cloud fractions from a ground-based whole-sky camera and MODIS were compared with ground-based LIDAR measurements. This study also suggested that we make better comparison for a same cloud field between satellite and multiple ground-based wholesky cameras. The findings of these studies indicate that the shipboard observations will contribute to the validation of cloud fractions for future satellite missions.

\section{Discussion}

In this section, we discuss the influence of ship fluctuations and the coefficient $k$ in Eq. (3).

In terms of the ship fluctuation, this study found that during the relevant period of JARE 55, the absolute values of pitch and roll were $9.84^{\circ}$ and $11.83^{\circ}$, respectively, between Australia and the sea ice region. As described in subsection 3.1, we restricted the available zenith angle to less than $70^{\circ}$ in every image so that we did not use the area within $20^{\circ}$ of the edge of the image in the analysis. Furthermore, the ceilometer measured the cloud base heights in the zenith direction based on the ship fluctuation, similar to the whole-sky camera. Consequently, we think the ship fluctuation did not substantially affect the results in this study even though we did not perform a fluctuation calibration based on the inclination of the vessel.

With respect to the validity of classification, we examined the sensitivity of the cloud fraction to coefficient $k$ in Eq. (3) and we summarized this in Table 3 . From this table, it is easily seen that the cloud fraction varies greatly depending on coefficient $k$. The cloud fraction has a considerable gap at around $k=5$, in particular, which suggests that some difference of coefficient $k$ (by one, for example) possibly leads to the severe bias in the estimation of the cloud fraction.

Furthermore, we investigated the sensitivity of coefficient $k$ to solar elevation, because the $S I-B I$ diagram in Fig. 6 depends on the brightness and color of the individual pixels. We selected by visual inspection those images between 14:00 UTC on 27 November 2013 and 13:30 UTC on 10 January 2014 that had 
Table 3. Sensitivity of the cloud fraction to coefficient $k$ in Eq. (3) for the image obtained at 06:15:19 UTC on 1 December 2013.

\begin{tabular}{cccc}
\hline$k$ & Cloud fraction $(\%)$ & $k$ & Cloud fraction $(\%)$ \\
\hline 2 & 99.1 & 6 & 2.4 \\
3 & 62.2 & 7 & 1.2 \\
4 & 44.7 & 8 & 0.8 \\
5 & 17.5 & 9 & 0.5 \\
\hline
\end{tabular}

no cloud. The period corresponds to the start of the ceilometer operation until the end of the whole-sky camera observations. As a result, we obtained 461 images with which to determine the blue sky coefficient $k_{b}$. Figure 11 shows the sensitivity of coefficient $k_{b}$ to the sun height for blue sky pixels. From Fig. 11 , it can be found that coefficient $k_{b}$ generally decreases as the sun height increases. Coefficient $k_{b}$ decreases rapidly with sun height from $0^{\circ}$ to $10^{\circ}$, whereas it changes little for the sun elevations higher than $10^{\circ}$. The sensitivity curves for the open ocean and sea ice field are clearly distinguished by the blue and pink plots, respectively, in Fig. 11.

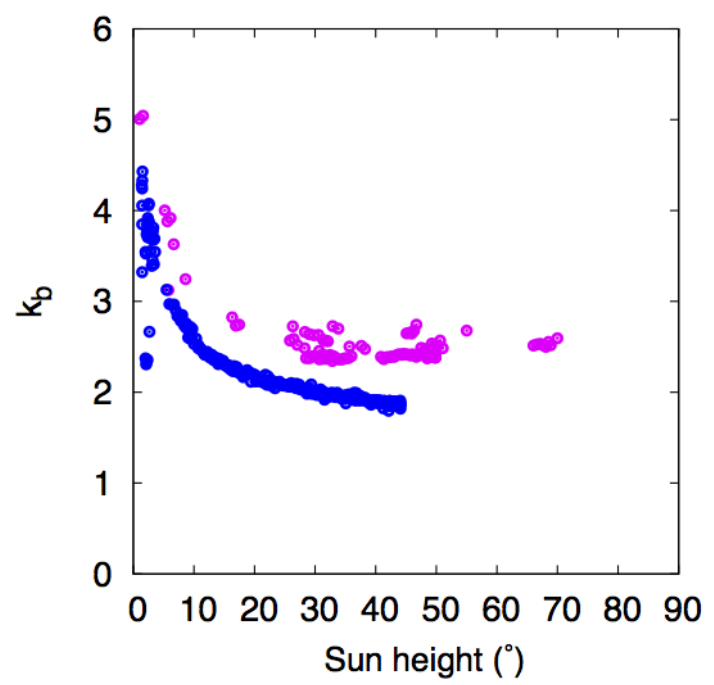

Fig. 11. Sensitivity of coefficient $k$ to the sun height $\left({ }^{\circ}\right)$ based on the 461 clear sky images from 14:00 UTC on 27 November 2013 to 13:30 UTC on 10 January 2014. The pink and blue plots correspond to the open ocean and the sea ice field, respectively.

We also selected overcast images by visual inspection for the same period as the clear sky images. As a result, we obtained 1588 images with the sun elevation higher than $10^{\circ}$, because we could not identify the darker images clearly with lower sun heights. Figure 12 shows the sensitivity of coefficient $k_{c}$ to the sun height for the overcast pixels. It can be seen that coefficient $k_{c}$ decreases linearly as the sun height increases. Coefficient $k_{c}$ has a larger vertical spread for each sun height compared with coefficient $k_{b}$, which seems to depend on cloud properties such as optical thickness and spatial distribution.

Coefficients $k_{b}$ and $k_{c}$ were at most three and at least four, respectively, at sun elevations higher than $10^{\circ}$ for the open ocean (pink plots in Figs. 11 and 12). It is reasonable, therefore, to claim that we have determined coefficient $k$ to be 3.7 with Eq. (4) in this preliminary analysis.

The $S I-B I$ method is considered a general classification approach in the spectral and brightness domain based on the JPEG information, as in other similar methods. ${ }^{7), ~ 8) ~ H o w e v e r, ~}$

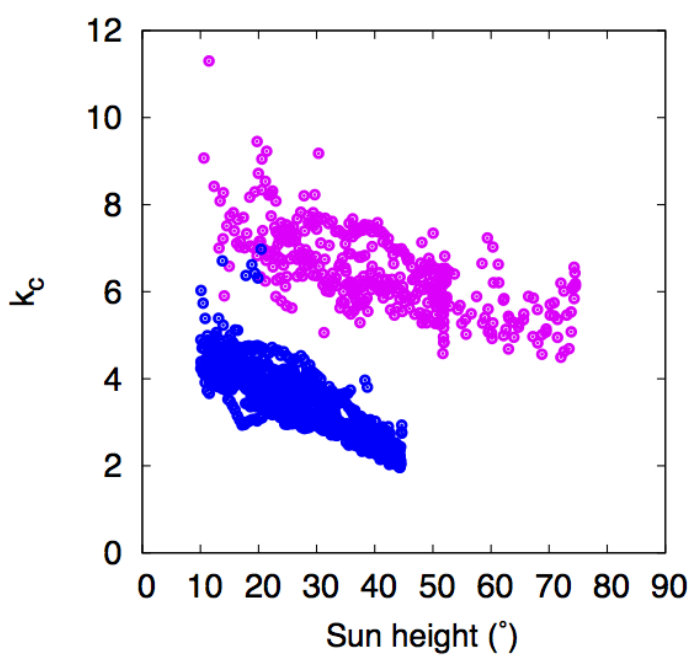

Fig. 12. Same as Fig. 11 based on 1588 cloudy images. The analysis was restricted to sun elevation higher than $10^{\circ}$ owing to difficulties related to image selection.

we might have an advantage in applying the algorithm to an imager over the ocean compared with over land because the $B I$ values are relatively smaller away from the solar point; i.e., $(S I, B I)=(0,1)$. This is because the cloud and sky brightnesses are relatively lower over the ocean because of lower surface albedo compared with land. The spectral contrast between cloud and blue sky pixels is clearer in the $S I$ domain because the pristine condition over the ocean, that is, relatively lower aerosol loading compared with land mean the path radiance is smaller. Accordingly, we might have more distinct clusters of sun, cloud, and blue sky pixels in the $S I-B I$ domain over the ocean compared with land, which possibly resulted in the robust algorithm in this study.

Finally, we discuss the consistency of the cloud fractions between the whole-sky camera and the ceilometer in the analyses. In addition to Fig. 10 and Table 2, we use the User's and Producer's Accuracies of the cloud fractions between Australia and Antarctica from 27 November to 9 December 2013 shown in Table 4.

From Table 4, it can be found that both accuracies are relatively high for cloud fraction of $95-100 \%$ (almost overcast): the User's Accuracy is $97.2 \%$ and the Producer's accuracy is $76.2 \%$. However, the accuracies show a different tendency for less cloudy situations. For example, the Producer's accuracies are $62.3 \%$ and $86.2 \%$ for cloud fractions of $0-5 \%$ and $5-95 \%$, respectively, while the User's Accuracies are $82.8 \%$ and $34.1 \%$, respectively. This relationship suggests that for smaller cloud fractions of $0-5 \%$, the ceilometer is more sensitive to optically thinner clouds than the whole-sky camera, whereas for moderate cloud fractions of 5-95\%, the ceilometer possibly dismisses clouds because of its narrower field of view. It is noted that the two remote sensors have quite different view angles: the wholesky camera has a wider view of $70^{\circ}$ from the zenith, while the ceilometer points only in the zenith direction. It will be necessary to consider the same fields of view when we compare cloud fractions derived from two imagers such as ground-based and spaceborne sensors. ${ }^{7), 8)}$ 
Table 4. User's and Producer's Accuracies of the cloud fractions between the whole-sky camera and the ceilometer. Cloud fraction was categorized in three bins: $0-5 \%, 5-95 \%$, and $95-100 \%$. The numbers of images are tabulated for each categories. Figures on the diagonal are shown in bold for clarity.

\begin{tabular}{ccccccc}
\hline & & \multicolumn{5}{c}{ Ceilometer } \\
\cline { 3 - 7 } & $\begin{array}{c}\text { Cloud } \\
\text { fraction \% }\end{array}$ & $0-5$ & $5-95$ & $95-100$ & Sum & $\begin{array}{c}\text { User's } \\
\text { Accuracy \% }\end{array}$ \\
\hline Whole- & $0-5$ & $\mathbf{1 4 4}$ & 30 & 0 & 174 & 82.8 \\
sky & $5-95$ & 87 & $\mathbf{2 3 2}$ & 362 & 681 & 34.1 \\
camera & $95-100$ & 0 & 7 & $\mathbf{1 1 6 2}$ & 1169 & 97.2 \\
& Sum & 231 & 269 & 1527 & 2024 & \\
& Producer's & 62.3 & 86.2 & 76.2 & & \\
& Accuracy \% & & & & & \\
\hline
\end{tabular}

\section{Concluding Remarks}

We made shipboard observation using a whole-sky camera and a ceilometer around Japan in the autumn of 2013 and during a round-trip cruise between Japan and Antarctica from November 2013 to April 2014. We analyzed the observational data to produce preliminary estimations of the cloud fractions between Australia and Antarctica from November to December 2013. Based on the results, it was found that the estimated cloud fractions were generally in agreement with each other, although the whole-sky camera underestimated the cloud fraction compared with the ceilometer. Future work should investigate this difference in detail in order to improve the retrieval of cloud fractions. We have identified several other issues to be considered in future research. First, the surface albedo variation should be taken into account, particularly over the sea ice region. Second, it might be possible to derive an alternative formulation of the classification curve other than the exponential type. Third, it would be constructive to implement edge detection techniques between cloud and clear sky based on the spatial contrast, in addition to the spectral contrast used in this study. Finally, an observational strategy could be devised for a whole-sky camera, in which the exposure time could be adjusted depending on the sun elevation, even if the diaphragm were fixed at a given value. The annual observational cruises with R/V Shirase will continue, and therefore the derived cloud fractions will contribute to the validation over ocean of satellite missions such as the GCOM-C/SGLI.

\section{Acknowledgments}

The authors are grateful to those who performed the observations onboard R/V Shirase during the 55th Japanese Antarctic Research Expedition (JARE 55). The authors are also grateful to Prof. Toshiyuki Murayama of Tokyo University of Marine Science and Technology, Japan, for his helpful comments, particularly with reference to the ceilometer measurements. The authors are also grateful to Mr. Kyohei Yamada of Tohoku University for his comments regarding the data analyses. This research was partially supported by a Global Change Observation Mission (GCOM; PI No. 204) project of the Japan Aerospace Exploration Agency (JAXA).

\section{References}

1) Smirnov, A., Holben, B. N., Slutsker, I., Giles, D. M., McClain, C. R., Eck, T. F., Sakerin, S. M., Macke, A., Croot, P., Zibordi, G., Quinn, P. K., Sciare, J., Kinne, S., Harvey, M., Smyth, T. J., Piketh, S., Zielinski, T., Proshutinsky, A., Goes, J. I., Nelson, N. B., Larouche, P., Radionov, V. F., Goloub, P., Krishna Moorthy, K., Matarrese, R., Robertson, E. J. and Jourdin, F.: Maritime Aerosol Network as a Component of Aerosol Robotic Network, Journal of Geophysical Research: Atmospheres, 114 (2009), pp.1-10.

2) Lyamani, H., Valenzuela, A., Perez-Ramirez, D., Toledano, C., Granados-Muñoz, M. J., Olmo, F. J. and Alados-Arboledas, L.: Aerosol Properties over the Western Mediterranean Basin: Temporal And Spatial Variability, Atmospheric Chemistry and Physics, 15 (2015), pp.2473-2486.

3) Brückner, M., Pospichal, B., Macke, A. and Wendisch, M.: A New Multispectral Cloud Retrieval Method for Ship-Based Solar Transmissivity Measurements, Journal of Geophysical Research: Atmospheres, 119 (2014), pp.11338-11354.

4) Heinle, A., Macke, A., and Srivastav, A.: Automatic Cloud Classification of Whole Sky Images, Atmos. Meas. Tech., 3 (2010), pp.557567.

5) Yabuki, M., Shiobara, M., Nishinaka, K. and Kuji, M.: Development of a Cloud Detection Method from Whole-Sky Color Images, Polar Science, 8 (2014), pp.315-326.

6) Yoshimura M. and Yamashita M.: Contribution of Ground-Based Cloud Observation to Satellite-Based Cloud Discrimination, J. Environ. Sci. Eng., A2 (2013), pp.379-382.

7) Letu, H., Nagao, T. M., Nakajima, T. Y. and Matsumae, Y.: Method for Validating Cloud Mask Obtained from Satellite Measurements Using Ground-Based Sky Camera, Applied Optics, 53 (2014), pp.7523-7533.

8) Katagiri, S., Hayasaka, T. and Yamada, K.: An Algorithm for a Whole-Sky Camera with Low Color Depth And Relationships between Cloud Fractions Derived from the Sky Camera And MODIS Observations, J. Remote Sensing Society Japan, 35 (2015), pp.89103. 